\title{
Article
}

\section{Flow of viscous fluid over an infinite plate with Caputo-Fabrizio derivatives}

\author{
M. Umar Farooq ${ }^{1}$, M. Saqib Khan ${ }^{1, *}$ and Ahmad Hajizadeh ${ }^{2,3}$ \\ 1 Department of Mathematics, Lahore Leads University, Lahore, 54600, Pakistan.; umaruet@yahoo.com (M.U.F); \\ khan.saqib@me.com(M.S.K) \\ 2 FAST, University Tun Hussein Onn Malaysia, 86400, Parit Raja, Batu Pahat, Johor State, Malaysia.; \\ as.zada@paaet.edu.kw \\ 3 Public Authority of Applied Education and Training, College of Technological Studies, Applied Science Department, \\ Shuwaikh, Kuwait. \\ * Correspondence: khan.saqib@me.com; Tell: +923214422580
}

Received: 1 March 2019; Accepted: 30 March 2019; Published: 24 April 2019.

\begin{abstract}
This paper presents Caputo-Fabrizio fractional derivatives approach to analysis of a viscous fluid over an infinite flat plate together with general boundary motion. Closed form exact general solutions of the fluid velocity are obtained by means of the Laplace transform. The solutions of ordinary viscous fluids corresponding to time-derivatives of integer order is obtained as particular cases of the present solutions. Several special cases are also discussed. Numerical computations and graphical illustrations are used in order to study the effects of the Caputo-Fabrizio time-fractional parameter $\alpha$ and Reynolds number on velocity field.
\end{abstract}

Keywords: Viscous fluid, Caputo-Fabrizio fractional derivatives, infinite plate, general boundary condition, closed form solution.

MSC: 05 C69.

\section{Introduction}

$\mathbf{T}$ he concept of fractional order derivatives is as old as for integer order derivatives. For the past three decades, this subject was limited only to mathematics. However, in the last few years, the concepts of fractional calculus were frequently applied to other disciplines. Recently, this subject has been extended in various directions such as fractional-order multipoles in electromagnetism, electrochemistry, tracer in fluid flows, model of neurons in biology, finance, signal processing, applied mathematics, bio engineering, viscoelasticity, fluid mechanics and fluid dynamics [1]. In fluid dynamics, the fractional derivative models are used widely for the study of viscoleastic materials such as polymers in the glass transition and glassy state [2]. Recently, it is increasingly seen as an efficient tool through which useful generalization of physical concept can be obtained. The most used fractional derivatives are the Riemann-Liouville fractional derivative and the Caputo fractional derivative [3-7]. It is known that, these operators exhibit difficulties in applications. For example, the Riemann-Liouvile derivative of a constant is not zero and, the Laplace transform of the Riemann-Liouville derivative contains terms without physical signification. The Caputo fractional derivative has eliminated these difficulties, but, the kernel of definition is a singular function. Caputo and Fabrizio have introduced recently, a new definition of the fractional derivatives with an exponential kernel without singularities [8]. The Caputo-Fabrizio temporal-fractional derivative is suitable to use the Laplace transform. The spatial representation of the Caputo-Fabrizio derivative is adequate to use the Fourier transform.

The aim of this report is to use the definition of Caputo-Fabrizio fractional derivative in order to obtain exact general solutions for the flow of an incompressible fractional viscous fluid over an infinite plate that is moving in its plane. The solutions that have been obtained are presented in simple forms and can be easily reduced to the similar solutions for ordinary fluids. Also compression of fractional and ordinary fluid are presented numerically and graphically. Rest of the paper is arranged as follows. The mathematical formulation of the problem is given in Section 2. Exact solutions via Laplace transform are established in Section 3 followed 
by some limiting cases in Section 4 and special cases in Section 5. Graphical results are portrayed in Section 6. This paper ends with some important conclusion in Section 7. Some important formulas used in this paper are presented in Appendix.

\section{Mathematical formulation of the problem}

We consider an incompressible viscous fluid occupying the space over an infinite plate which is situated in the $(x, z)$-plane of the Cartesian coordinate system with the positive $y$-axis in the upward direction. Initially, both the fluid and the plate are at rest. At $t=0^{+}$, the fluid is set in motion by the plate, which begins to move along the $x$-axis. The velocity of the plate is assumed to be of the form $U_{0} f(t)$, where $U_{0}>0$ is a constant and $f(t)$ is a piecewise continuous function defined on $[0, \infty)$ and $f(0)=0$. Furthermore, we suppose that the Laplace transform of the function $f(t)$ exists. In the case of parallel flow along the $x$-axis, the velocity vector is $V=u(y, t), 0,0$ leads us to the following governing equations:

$$
\begin{aligned}
\frac{\partial u(y, t)}{\partial t} & =v \frac{\partial^{2} u(y, t)}{\partial y^{2}} \\
\tau(y, t) & =\mu \frac{\partial u(y, t)}{\partial y}
\end{aligned}
$$

where $v$ is the kinematic viscosity, $\tau(y, t)=S_{x y}(y, t)$ is the nonzero shear stress, $\mu$ is the dynamic viscosity of the fluid. The adequate initial and boundary conditions are given by

$$
u(y, 0)=0 ; y \geq 0, u(0, t)=U_{0} f(t), t>0 u(y, t) \rightarrow 0, \text { as } y \rightarrow \infty .
$$

Introducing the following non-dimensional quantities to equations (1), (2) and (3); $t^{*}=\frac{t}{T} ; T>0, y^{*}=\frac{y}{u_{0} T}, u^{*}=\frac{u}{u_{0}}, \tau^{*}=\frac{\tau}{\rho u_{0}^{2}}, f^{*}\left(t^{*}\right)=f\left(T t^{*}\right)$, and dropping out the ${ }^{\prime * \prime}$ notation, we get the following non-dimensional problem:

$$
\begin{aligned}
\frac{\partial u(y, t)}{\partial t} & =\frac{1}{\operatorname{Re}} \frac{\partial^{2} u(y, t)}{\partial y^{2}} \\
\tau(y, t) & =\frac{1}{\operatorname{Re}} \frac{\partial u(y, t)}{\partial y} .
\end{aligned}
$$

The non-dimensional initial and boundary conditions are

$$
\begin{array}{rlrl}
u(y, 0) & =\frac{\partial u(y, 0)}{\partial t}=0, & & y \geq 0, \\
u(0, t) & =f(t), & & \text { for } t>0, \\
u(y, t) \rightarrow 0, & \text { as } y \rightarrow \infty
\end{array}
$$

where $\operatorname{Re}=\frac{U_{0}^{2} T}{v}$ is the Reynolds number.

In order to develop a model with time-fractional derivatives, we replace the first order time derivative with Caputo-Fabrizio time-fractional derivative of order $\alpha \in 0,1$. Thus, (4) is written as,

$$
D_{t}^{\alpha} u(y, t)=\frac{1}{\operatorname{Re}} \frac{\partial^{2} u(y, t)}{\partial y^{2}}
$$

where Caputo-Fabrizio time-fractional derivative [8] is defined as,

$$
D_{t}^{\alpha} u(y, t)=\frac{1}{1-\alpha} \int_{0}^{t} \exp \left[\frac{-\alpha(t-s)}{1-\alpha}\right] u^{\prime}(y, s) d s, \quad 0<\alpha<1 .
$$




\section{Calculation for velocity field}

If we apply the Laplace transform to equation (9) and conditions (7), (8) and use the initial conditions, we get the following.

$$
\begin{aligned}
\frac{\partial^{2} \bar{u}(y, q)}{\partial y^{2}} & =\frac{\operatorname{Re} \gamma q}{q+\alpha \gamma} \bar{u}(y, q) \\
\bar{u}(0, q) & =F(q) \\
\bar{u}(y, t) & \rightarrow 0, \text { as } y \rightarrow \infty
\end{aligned}
$$

where $\bar{u}(y, q)$ and $F(q)$ are the Laplace transform of $u(y, t)$ and $f(t)$ respectively, and ' $q$ ' is the transform variable.

The solution of the partial differential equation (11) by using conditions (12) and (13) is given as

$$
\bar{u}(y, q)=F(q) \exp \left[-y \sqrt{\frac{\operatorname{Re} \gamma q}{q+\alpha \gamma}}\right] .
$$

Similarly, (14) can be written in the equivalent form as,

$$
\bar{u}(y, q)=q F(q) \frac{1}{q} \exp \left[-y \sqrt{\frac{\operatorname{Re} \gamma q}{q+\alpha \gamma}}\right] .
$$

Applying the inverse Laplace transform and using convolution product, we obtain

$$
u(y, t)=\int_{0}^{t} f^{\prime}(t-s) \phi(y, s ; \operatorname{Re} \gamma, \alpha \gamma) d s,
$$

where $f^{\prime}(t)=L^{-1}\{q F(q)\}$ and $\phi(y, t ; a, b)=L^{-1}\{\Phi(y, q ; a, b)\}$ is defined in Appendix (A1) and (A2).

\section{Velocity field to ordinary viscous fluid case $(\alpha=1)$}

The velocity field, corresponding to the ordinary case of $\alpha=1$, is obtained by using the properties of Caputo-Fabrizio fractional derivative.

Consider the following.

$$
\lim _{\alpha \rightarrow 1} \phi(y, t ; \operatorname{Re} \gamma, \alpha \gamma)=\lim _{y \rightarrow \infty} \Phi(y, t ; \operatorname{Re} \gamma, \alpha \gamma)=1-\frac{2}{\pi} \int_{0}^{\infty} \frac{\sin (y x)}{x} \exp \left\{-\frac{t x^{2}}{\operatorname{Re}}\right\} d x
$$

Using (10), we get,

$$
\int_{0}^{\infty} \frac{\sin (b x)}{x} \exp \left(-a x^{2}\right) d x=\frac{\pi}{2} \operatorname{erfc}\left\{\frac{b}{2 \sqrt{a}}\right\}
$$

we obtain

$$
\lim _{\alpha \rightarrow 1} \phi\{y, t ; \operatorname{Re} \gamma, \alpha \gamma\}=1-\operatorname{erfc}\left\{\frac{y \sqrt{\operatorname{Re}}}{2 \sqrt{t}}\right\}=\operatorname{erfc}\left\{\frac{y \sqrt{\operatorname{Re}}}{2 \sqrt{t}}\right\},
$$

which gives the velocity field as follows.

$$
u(y, t)=\int_{0}^{t} h\{t-s\} \operatorname{erfc}\left\{\frac{y \sqrt{\operatorname{Re}}}{2 \sqrt{s}}\right\} d s
$$

\section{Special Cases}

In order to discuss the theoretical and practical value of general solutions and to certify their accuracy or to gain some physical insight for some motions with technical relevance, three special cases are considered in the following. Actually, these general solutions can provide the dimensionless velocity field corresponding to any motion problem of this type of fluids. 


\subsection{Plate moves with constant velocity: $\{f(t)=H(t)\}$}

Let $H(\cdot)$ be the Heaviside unit step function. Then, the dimensionless velocity field, representing the motion induced by an infinite plate that is moving in its plane, with a constant velocity is as follows.

$$
u(y, t)=\int_{0}^{t} \delta(t-s) \phi\{y, s ; \operatorname{Re} \gamma, \alpha \gamma\} d s=\phi\{y, t ; \operatorname{Re} \gamma, \alpha \gamma\}
$$

5.2. Plate moves with oscillating velocity: $\{f(t)=\sin (t)\}$

The dimensionless velocity field, representing the solution corresponding to motions due to a plate that is oscillating in its plane, is given by,

$$
u(y, t)=\int_{0}^{t} \cos \{t-s\} \phi\{y, s ; \operatorname{Re} \gamma, \alpha \gamma\} d s .
$$

5.3. Plate moves with exponential velocity: $\{f(t)=H(t) \exp (a t)\}$

The dimensionless velocity field is

$$
u(y, t)=\phi(y, t ; \operatorname{Re} \gamma, \alpha \gamma)+\psi(y, t ; \operatorname{Re} \gamma, \alpha \gamma, c)
$$

where $\psi(y, t ; a, b, c)$ is defined in Appendix (A3).

\section{Numerical results and discussions}

In order to obtain some information on the fluid flow parameters, we have made several numerical simulations using Mathcad software. Obtained results are presented in the Figures $1-3$. All the parameters and profiles are dimensionless. For curves we consider the function $f(t)=H(t)$. We are interested, first, to analyze the influence of the fractional parameter, $\alpha$, on the dimensionless fluid flow velocity. Also, the influence of the Reynolds number, Re, on the fluid velocity were studied. The ordinary fluid, corresponding to the unit fractional parameter, is compared with few cases with the fractional parameter. The Figures 1 and 2 are plotted in order to discuss the influence of the fractional parameter $\alpha$ on the fluid velocity. The curves corresponding to fluid velocity $u(y, t)$, are sketched versus $y$, for small and large values of time and of fractional parameter $\alpha$, namely, $\alpha \in\{0.4,0.6,0.8,1.0\}$. For Reynolds number, we used the value $\operatorname{Re}=3$. The Figure 1 is plotted for small time $t$. It is observed that by increasing values of the fractional parameter $\alpha$, the fluid velocity decreases and by increasing the time $t$, the difference between the fluid velocities decreases. Figure 2 is plotted for large time $t$. It is observed that it has opposite behavior from Figure 1. The influence of Reynolds number on the fluid velocity is shown in Figure 3. For curves plotted in Figure 3, the fractional model corresponding to $\alpha=0.5$ was considered. It is observed from Figure 3 that, at small values of Reynolds number, the velocity is larger. It is also observed that for small and large values time $t$, the behavior is same only by increasing the values of time $t$, the difference between the velocities is significant.
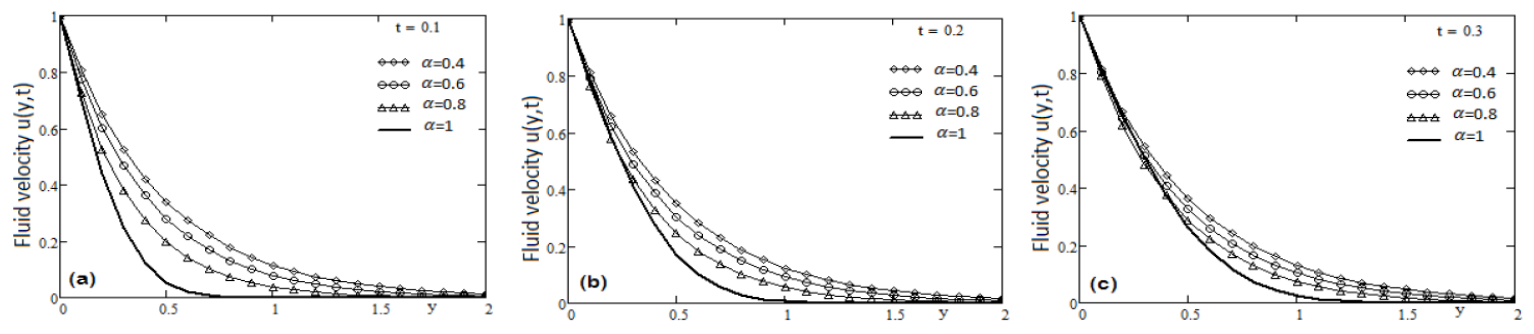

Figure 1. Profiles of velocities for fractional parameter variation for different small time and fixed Reynolds number $\operatorname{Re}=3$. 

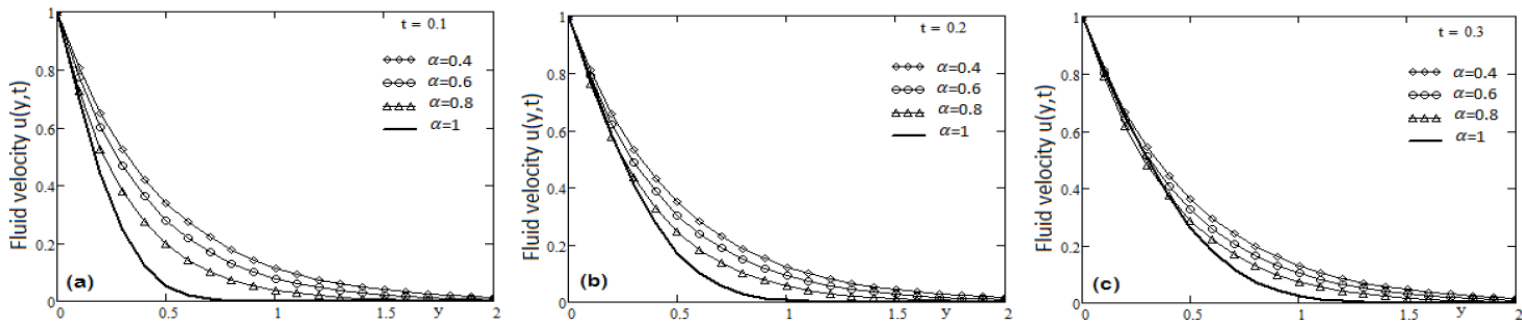

Figure 2. Profiles of velocities for fractional parameter variation for different large time and fixed Reynolds number $\operatorname{Re}=3$.
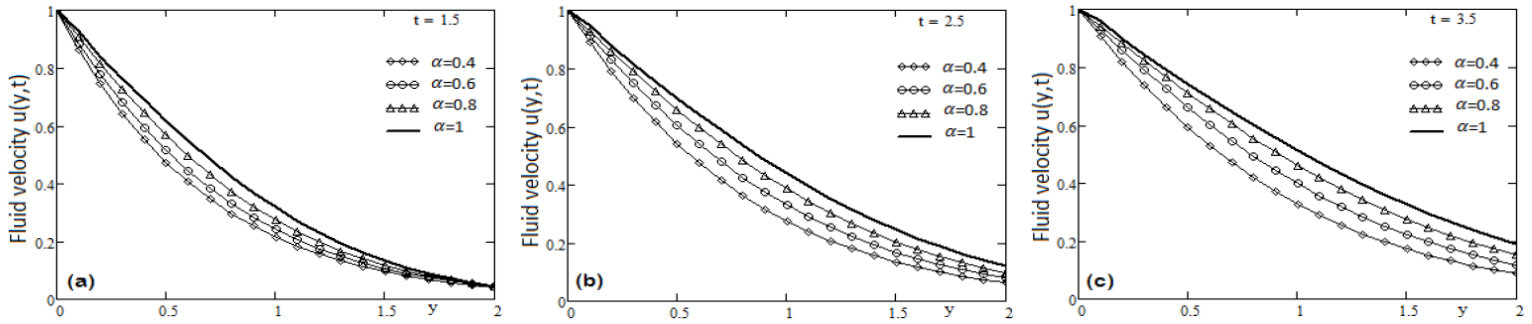

Figure 3. Profiles of velocities for Reynolds number variation for different time and fixed fractional parameter $\alpha=0.5$.

\section{Conclusions}

We presented a model of Newtonian fluid with Caputo-Fabrizio fractional derivatives, over an infinite flat plate together with general boundary motion. Closed form exact general solutions of the fluid velocity are obtained by means of the Laplace transform. The following are observations:

1. It is observed that by increasing values of the fractional parameter, the fluid velocity decreases.

2. By increasing the time $t$, the difference between the fluid velocities decreases.

3. At small values of Reynolds number, the velocity is larger.

4. It is also observed that for small and large values of time $t$, the behavior is same only by increasing the values of time $t$, the difference between the velocities is significant.

\section{Appendix}

$$
\begin{gathered}
\Phi(y, s ; a, b)=\frac{1}{s} \exp \left\{-y \sqrt{\frac{a s}{s+b}}\right\} . \\
\phi(y, t ; a, b)=L^{-1}(\Phi(y, s ; a, b))=1-\frac{2 a}{\pi} \int_{0}^{\infty} \frac{\sin (y x)}{x\left\{a+x^{2}\right\}} \exp \left\{-\frac{b t x^{2}}{a+x^{2}}\right\} d x . \\
\psi(y, t ; a, b, c)=\exp \left\{c t-y \sqrt{\frac{a c}{b+c}}\right\}-1-\frac{2 a c}{\pi} \int_{0}^{\infty} \frac{\sin (y x)}{x\left\{a c+(b+c) x^{2}\right\}} \exp \left(-\frac{b t x^{2}}{a+x^{2}}\right) d x .
\end{gathered}
$$

Author Contributions: All authors contributed equally to the writing of this paper. All authors read and approved the final manuscript.

Conflicts of Interest: "The authors declare no conflict of interest."

\section{References}

[1] Kulish, V. V., \& Lage, J. L. (2002). Application of fractional calculus to fluid mechanics. Journal of fluids engineering, 124(3), 803-806.

[2] Debnath, L. (2003). Recent applications of fractional calculus to science and engineering. International Journal of Mathematics and Mathematical Sciences, 54, 3413-3442. 
[3] Hilfer, R. (2008). Threefold introduction to fractional derivatives. Anomalous Transport: Foundations and Applications, Weinheim: Wiley-VCH Verlag.

[4] Gorenflo R., Mainardi F., Moretti D. and Paradisi P. (2002) Time fractional diffusion: a discret random walk approach. Nonlinear Dynam. 29, 129-143, DOI: 10.1023/A:1016547232119.

[5] Mainardi, F. (2010). Fractional calculus and waves in linear viscoelasticity. An introduction to mathematical models, World Scientific Publishing.

[6] Podlubny, I. (2009). Fractional differential equations. Academic Press, New York.

[7] Caputo, M., \& Fabrizio, M. (2015). Damage and fatigue described by a fractional derivative model. Journal of Computational Physics, 293, 400-408.

[8] Caputo, M., \& Fabrizio, M. (2015). A new definition of fractional derivative without singular kernel. Progress in fractional differentiation and Applications, 1(2), 1-13.

[9] Gradshteyn, I. S., \& Ryzhik, I. M. (2014). Table of integral, series and products. Eighth Ed., Academic Press, USA.

(C) 2019 by the authors; licensee PSRP, Lahore, Pakistan. This article is an open access article distributed under the terms and conditions of the Creative Commons Attribution (CC-BY) license (http://creativecommons.org/licenses/by/4.0/). 